\title{
Chemokines in myocardial infarction: translating basic research into clinical medicine
}
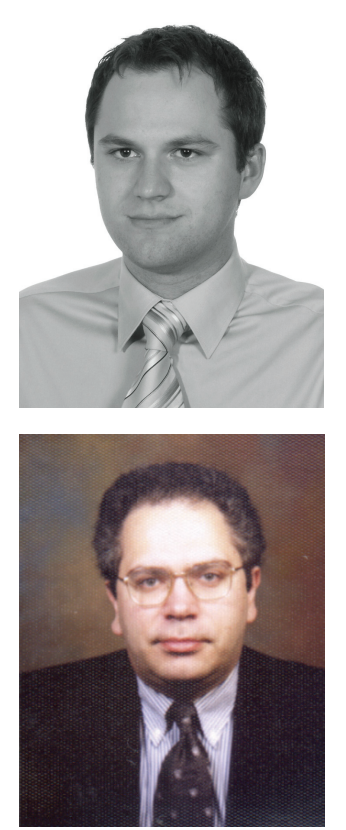

Marcin Dobaczewski \& Nikolaos $G$

Frangogiannis ${ }^{\dagger}$

${ }^{\dagger}$ Author for correspondence Baylor College of Medicine, Section of Cardiovascular Sciences, One Baylor Plaza M/S F-602, Houston, TX 77030, USA

Tel.: + 17137984188 ; Fax: +17137960015 ; ngf@bcm.tmc.edu

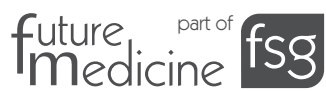

\author{
'Our goal is no longer to \\ nonspecifically inhibit inflammation \\ in order to reduce 'inflammatory \\ injury', but rather to modulate \\ carefully selected cellular and \\ molecular mediators of \\ inflammation in order to promote \\ optimal cardiac repair.'
}

Despite the decreasing incidence of coronary artery disease, myocardial infarction remains a major clinical problem and a significant cause of morbidity and mortality in western societies. In 2006, an estimated 565,000 Americans had a first myocardial infarction, while 300,000 had a recurrent event. Morbidity and mortality after the acute phase of myocardial infarction often results from cardiac failure owing to structural and functional alterations of the healing infarcted ventricle. Postinfarction cardiac repair is closely intertwined with an inflammatory cascade that ultimately results in formation of a scar. Infarct healing is associated with profound changes in ventricular architecture and geometry, also referred to as 'ventricular remodeling', a complex and poorly understood process that results in chamber dilation, systolic and diastolic dysfunction, and development of a spherical geometry [1]. Ventricular dilation after myocardial infarction is linked to heart failure progression and is an important predictor of mortality and adverse cardiac events [2].

'Recent experiments using genetically targeted animals demonstrated the important role of the MCP-1/CCR2 axis in the regulation of infarct healing...'

Although recent advances in cardiovascular therapy have resulted in the development of novel therapeutic strategies that salvage myocardium and improve early mortality in patients with acute myocardial infarction, approaches directly targeting the healing process are lacking. Development of therapeutic interventions attenuating postinfarction remodeling requires understanding of the mechanisms involved in regulation of the inflammatory and fibrotic response following myocardial infarction [3,4].

\section{Biology of the chemokines}

The chemokines comprise a family of small (8-14 kDa) highly basic proteins with a strikingly similar tertiary structure [5]. They have been divided into subfamilies on the basis of the number and sequential relationship of their conserved cysteine residues (CXC, CC, XC and $\mathrm{CX}_{3} \mathrm{C}$ subfamilies). CC chemokines are the most numerous and diverse family, including at least 25 ligands in humans. CXC chemokines are further classified according to the presence of the tripeptide motif glutamic acid-leucine-arginine (ELR) in the aminoterminal region [6].

Chemokines play a critical role in basal and inflammatory leukocyte locomotion and trafficking $[7,8]$ and their principal targets are bone marrow-derived cells. In addition to effects on cell locomotion, certain chemokines are capable of eliciting a variety of other responses affecting leukocyte adhesion [9], activation and degranulation, mitogenesis and apoptosis. Chemokines also exert important actions on many cell types beyond the immune system, including endothelial cells (resulting in angiogenic or angiostatic effects) [10], smooth muscle cells, neurons and epithelial cells.

Investigations using animal models of myocardial infarction have demonstrated that several members of the chemokine family are rapidly and consistently induced in the infarcted heart and suggested that they may play an important role in regulating the postinfarction inflammatory response [11-15]. The CC chemokines monocyte chemoattractant protein (MCP)-1/CCL2, macrophage inflammatory protein (MIP)-1 $\alpha /$ CCL3 and MIP-1 $\beta / C C L 4$ and the CXC chemokines IL-8/CXCL8, IFN- $\gamma$ inducible protein (IP)-10/CXCL10 and stromal cell-derived factor (SDF)-1/CXCL12 are markedly upregulated in the infarcted myocardium [16]. Recent experiments using genetically targeted animals demonstrated the important role of the MCP-1/CCR2 axis in the regulation of infarct 
healing and in the pathogenesis of cardiac remodeling. $\mathrm{MCP}-1 / \mathrm{CCL} 2^{-/}$mice had decreased and delayed macrophage infiltration and suppressed cytokine expression in the infarcted myocardium [17]. Both MCP-1 ${ }^{-/-}[17]$ and CCR2 $^{-/-}[18]$ mice exhibit attenuated ventricular dilation following myocardial infarction. In addition, anti-MCP-1 gene therapy reduced chamber dilation following infarction, attenuating systolic dysfunction and improving survival in a mouse model [19]. Although these findings provided the first evidence that chemokines may be novel therapeutic targets in patients with myocardial infarction, several important issues need to be addressed before attempting to translate knowledge derived from experimental studies into clinical therapy.

\section{MCP-1 \& SDF-1 as therapeutic targets in myocardial infarction}

Understanding the role of each chemokine in the complex process of infarct healing is crucial for the identification of potential therapeutic targets. Although chemokine induction is a prominent feature of the postinfarction inflammatory response, mechanistic information on the role of chemokine-mediated interactions is lacking. The increasing availability of genetically targeted mice and the development of antibodies that inhibit specific chemokines will likely result in the rapid growth of our knowledge in the field. At this stage, there is emerging evidence to support that inhibition of the MCP-1 pathway [20] and activation of SDF-1-mediated mechanisms may be promising therapeutic approaches in myocardial infarction.

\section{MCP-1 inhibition in postinfarction remodeling}

One of the best studied CC chemokines, MCP-1/CCL2, is a potent chemoattractant for mononuclear cells, and has been implicated in diseases characterized by monocyte-rich infiltrates [21,22].

'Although chemokine induction is
a prominent feature of the
postinfarction inflammatory response,
mechanistic information on the
role of chemokine-mediated
interactions is lacking.'

In addition to its critical role in mononuclear cell recruitment, MCP-1 exerts important actions on nonhematopoietic cells, inducing angiogenic and arteriogenic effects [23] and modulating fibroblast phenotype and activity [24]. In order to examine the role of MCP-1 in infarct healing, we studied the effects of MCP-1 gene disruption and antibody neutralization in a mouse model of myocardial infarction [17]. MCP-1 $1^{-/}$mice had decreased and delayed macrophage infiltration in the healing infarct and demonstrated delayed replacement of dead cardiomyocytes with granulation tissue. MCP- $1^{-/}$infarcts had decreased proinflammatory cytokine expression, exhibited attenuated levels of the inhibitory cytokines TGF- $\beta$, and IL-10 and demonstrated defective macrophage differentiation evidenced by decreased osteopontin (OPN)-1 expression. MCP-1 deficiency significantly diminished myofibroblast accumulation. Despite showing delayed phagocytotic removal of dead cardiomyocytes, $\mathrm{MCP}-1^{-/-}$mice had attenuated left ventricular remodeling, but similar infarct size, when compared with wildtype animals. MCP-1 antibody inhibition resulted in defects comparable with the pathological findings noted in infarcted MCP-1 $1^{-/}$animals without an effect on macrophage recruitment [17].

\section{$\therefore$...recruitment of CXCR4+ blood-derived progenitors through activation of chemokine-mediated pathways may be a promising therapeutic approach...'}

Our findings indicated that MCP-1 has important effects on macrophage recruitment and activation, cytokine synthesis and myofibroblast accumulation in healing infarcts. MCP-1 gene disruption results in decreased fibroblast infiltration and attenuated remodeling at the expense of a delayed inflammatory phase and impaired phagocytosis of dead cardiomyocytes. Thus, interventions targeting MCP- 1 may have beneficial effects by reducing ventricular dilation; however, they may also result in impaired and delayed clearance of the wound from dead cells. The clinical consequences of this defect cannot be safely predicted on the basis of experimental studies in rodents.

\section{SDF-1: a promising new strategy for tissue regeneration}

SDF-1/CXCL12 is a non-ELR containing CXC chemokine with chemotactic effects for $\mathrm{CD} 34^{+}$ progenitors [25] and primitive hematopoietic cells [26]. SDF-1 may regulate the recruitment, maturation and function of CXCR4-expressing 
progenitor cells in ischemic tissues [27,28]. Transplantation of cells engineered to express SDF-1 into the peri-infarct zone following coronary occlusion in the rat resulted in attenuated adverse remodeling [27]. The beneficial effects of SDF-1 may be due to therapeutic stem cell homing into the injured myocardium resulting in neovascularization and enhanced preservation of cardiomyocytes [29]. Local delivery of SDF-1 in the infarct may exert beneficial actions by augmenting vasculogenesis and by protecting ischemic cardiomyocytes from apoptosis without inducing cardiomyocyte regeneration. These findings suggest that enhanced recruitment of $\mathrm{CXCR}^{+}$blood-derived progenitors through activation of chemokine-mediated pathways may be a promising therapeutic approach in patients with myocardial infarction.

\section{Chemokine-related approaches in patients with myocardial infarction: challenges \& therapeutic opportunities}

Although experimental studies have identified MCP-1 and SDF-1 as potential therapeutic targets in myocardial infarction, several considerations need to be taken into account before translation of chemokine-related approaches into clinical therapy is attempted.

\section{'...postinfarction chemokine response and the healing defects exhibited by certain high-risk subgroups of patients, such as the elderly, diabetics and obese patients, need to be investigated.'}

First, the concepts derived from studies using rodents need to be validated in large animal models of myocardial infarction. There are some significant differences in chemokine-receptor expression and function between mice and humans. An example is that CCR1 is prominently expressed by mouse neutrophils, but has only limited expression in human neutrophils [30]. In addition, there is an occasional lack of homology between human and rodent chemokines. IL8/CXCL8, a key neutrophil chemoattractant in large mammals, does not have a structural homolog in rodents. In addition, the pathologic and electrophysiologic features of the infarcted heart in rodents exhibit significant differences when compared with large mammals and humans [31]. Thus, conclusions based on mouse or rat studies may not be directly relevant to the human pathobiology.
Second, many chemokines are pleiotropic and multifunctional agents, likely to modulate several distinct cellular processes in the infarcted myocardium. Thus, approaches expected to attenuate injury may prove deleterious owing to the modulation of important reparative pathways.

\section{'Activation of chemokine pathways may be important in the early \\ stages of healing in order to attract specific leukocyte subsets that clear the wound of dead cardiomyocytes and matrix fragments.'}

Timing and topography should be carefully considered when designing therapeutic strategies for patients with myocardial infarction. Effective healing is dependent on a well-orchestrated cellular response and on the timely induction and suppression of specific mediators in a locally restricted manner. Thus, interventions targeting inflammatory mediators should take into account both topographic and temporal parameters. An approach that may have favorable effects if locally applied in the center of the infarct may result in deleterious changes in the border zone or the noninfarcted remodeling myocardium. Furthermore, timing of an intervention is of critical significance. Activation of chemokine pathways may be important in the early stages of healing in order to attract specific leukocyte subsets that clear the wound of dead cardiomyocytes and matrix fragments. By contrast, prolonged chemokine upregulation as the wound matures, or extension of the chemokine response into the noninfarcted remodeling myocardium, may result in expansion of the inflammatory infiltrate and enhanced fibrosis in the viable areas [32].

Third, the local delivery of chemokines in the myocardium may be limited by rapid diffusion from the site of injection and by proteolytic degradation. For example, SDF-1 is cleaved by matrix metalloproteinase- 2 generating a neurotoxic remnant. Thus, the effectiveness of SDF-1 therapy may be limited in the inflammatory and protease-rich environment of the infarct. Design of protease-resistant chemokine molecules may be necessary for effective therapy [33].

The alterations in the postinfarction chemokine response and the healing defects exhibited by certain high-risk subgroups of patients, such as the elderly, diabetics and obese patients, need to be investigated. Senescent animals exhibit accentuated adverse remodeling associated with 
suppressed inflammation and formation of a defective scar with a low collagen content [34]. Diabetes is associated with enhanced ischemic injury and delayed repair, in part due to an attenuated and defective inflammatory response [35,36]. Impaired healing may be responsible for adverse outcome in these high-risk groups. Identification of specific defects in the regulation of chemokine signaling may suggest treatment strategies aimed at optimizing infarct healing in the most vulnerable patients.

The significant progress in our understanding of the pathobiology of the inflammatory response will greatly contribute to identification of therapeutic targets for patients with myocardial infarction. Our goal is no longer to nonspecifically inhibit inflammation in order to reduce 'inflammatory injury', but rather to modulate carefully selected cellular and molecular mediators of inflammation in order to promote optimal cardiac repair. Distinct leukocyte subsets may mediate divergent responses in the healing infarct; these subsets may be mobilized through distinct chemokine pathways. Thus, the chemokine system provides us with unique opportunities to selectively modulate the profile of bloodderived cells infiltrating the infarct in order to optimize the reparative process.

\section{Financial \& competing interests disclosure}

Nikolaos Frangogiannis' laboratory is supported by grants NIH R01 HL-76246 and NIH R01 HL-85440. The authors have no other relevant affiliations or financial involvement with any organization or entity with a financial interest in or financial conflict with the subject matter or materials discussed in the manuscript apart from those disclosed.

No writing assistance was utilized in the production of this manuscript.

\section{Bibliography}

1. Pfeffer MA, Braunwald E: Ventricular remodeling after myocardial infarction. Experimental observations and clinical implications. Circulation 81, 1161-1172 (1990).

2. St John Sutton M, Lee D, Rouleau JL et al.: Left ventricular remodeling and ventricular arrhythmias after myocardial infarction. Circulation 107, 2577-2582 (2003).

3. Frangogiannis NG: The mechanistic basis of infarct healing. Antioxid. Redox Signal. 8, 1907-1939 (2006).

4. Frangogiannis NG: Targeting the inflammatory response in healing myocardial infarcts. Curr. Med. Chem. 13, 1877-1893 (2006).

5. Clark-Lewis I, Kim KS, Rajarathnam K et al:: Structure-activity relationships of chemokines. J. Leukoc. Biol. 57, 703-711 (1995).

6. Luster AD: Chemokines - chemotactic cytokines that mediate inflammation. N. Engl. J. Med. 338, 436-445 (1998).

7. Gerard C, Rollins BJ: Chemokines and disease. Nat. Immunol. 2, 108-115 (2001).

8. Moser B, Loetscher P: Lymphocyte traffic control by chemokines. Nat. Immunol. 2, 123-128 (2001).

9. Gerszten RE, Garcia-Zepeda EA, Lim YC et al: : MCP-1 and IL-8 trigger firm adhesion of monocytes to vascular endothelium under flow conditions. Nature 398, 718-723 (1999).

10. Strieter RM, Polverini PJ, Arenberg DA et al:: Role of C-X-C chemokines as regulators of angiogenesis in lung cancer. J. Leukoc. Biol. 57, 752-762 (1995).
11. Frangogiannis NG: Chemokines in the ischemic myocardium: from inflammation to fibrosis. Inflamm. Res. 53, 585-595 (2004).

12. Kumar AG, Ballantyne CM, Michael LH et al:: Induction of monocyte chemoattractant protein-1 in the small veins of the ischemic and reperfused canine myocardium. Circulation 95, 693-700 (1997).

13. Frangogiannis NG, Mendoza LH, Lewallen M, Michael LH, Smith CW, Entman ML: Induction and suppression of interferon-inducible protein 10 in reperfused myocardial infarcts may regulate angiogenesis. FASEB J. 15, 1428-1430 (2001).

14. Kukielka GL, Smith CW, LaRosa GJ et al: Interleukin- 8 gene induction in the myocardium after ischemia and reperfusion in vivo. J. Clin. Invest. 95, 89-103 (1995).

15. Frangogiannis NG: Chemokines in ischemia and reperfusion. Thromb. Haemost. 97, 738-747 (2007).

16. Frangogiannis NG: The role of the chemokines in myocardial ischemia and reperfusion. Curr. Vasc. Pharmacol. 2, 163-174 (2004).

17. Dewald O, Zymek P, Winkelmann $\mathrm{K}$ et al.: CCL2/monocyte chemoattractant protein-1 regulates inflammatory responses critical to healing myocardial infarcts. Circ. Res. 96, 881-889 (2005).

18. Kaikita K, Hayasaki T, Okuma T, Kuziel WA, Ogawa H, Takeya M: Targeted deletion of CC chemokine receptor 2 attenuates left ventricular remodeling after experimental myocardial infarction. Am. J. Pathol. 165, 439-447 (2004).
19. Hayashidani S, Tsutsui H, Shiomi T et al.: Anti-monocyte chemoattractant protein-1 gene therapy attenuates left ventricular remodeling and failure after experimental myocardial infarction. Circulation 108, 2134-2140 (2003).

20. Xia Y, Frangogiannis NG: MCP-1/CCL2 as a therapeutic target in myocardial infarction and ischemic cardiomyopathy. Inflamm. Allergy Drug Targets 6, 101-107 (2007).

21. Rollins BJ: Monocyte chemoattractant protein 1: a potential regulator of monocyte recruitment in inflammatory disease. Mol. Med. Today 2, 198-204 (1996).

22. Gu L, Tseng SC, Rollins BJ: Monocyte chemoattractant protein-1. Chem. Immunol. 72, 7-29 (1999).

23. Salcedo R, Ponce ML, Young HA et al: Human endothelial cells express CCR2 and respond to MCP-1: direct role of MCP-1 in angiogenesis and tumor progression. Blood 96, 34-40 (2000).

24. Gharaee-Kermani M, Denholm EM, Phan SH: Costimulation of fibroblast collagen and transforming growth factor $\beta 1$ gene expression by monocyte chemoattractant protein-1 via specific receptors. J. Biol. Chem. 271, 17779-17784 (1996).

25. Aiuti A, Webb IJ, Bleul C, Springer T, Gutierrez-Ramos JC: The chemokine SDF-1 is a chemoattractant for human $\mathrm{CD} 34^{+}$hematopoietic progenitor cells and provides a new mechanism to explain the mobilization of $\mathrm{CD} 34^{+}$progenitors to peripheral blood. J. Exp. Med. 185, 111-120 (1997). 
26. Jo DY, Rafii S, Hamada T, Moore MA: Chemotaxis of primitive hematopoietic cells in response to stromal cell-derived factor-1. J. Clin. Invest. 105, 101-111 (2000).

27. Askari AT, Unzek S, Popovic ZB et al: Effect of stromal-cell-derived factor 1 on stem-cell homing and tissue regeneration in ischaemic cardiomyopathy. Lancet 362, 697-703 (2003).

28. Togel F, Isaac J, Hu Z, Weiss K, Westenfelder C: Renal SDF-1 signals mobilization and homing of CXCR4-positive cells to the kidney after ischemic injury. Kidney Int. 67, 1772-1784 (2005).

29. Zhang M, Mal N, Kiedrowski M et al.: SDF-1 expression by mesenchymal stem cells results in trophic support of cardiac myocytes after myocardial infarction. FASEB J. 21, 3197-3207 (2007).

30. Hancock WW, Gao W, Faia KL, Csizmadia V: Chemokines and their receptors in allograft rejection. Curr. Opin. Immunol. 12, 511-516 (2000).
31. Dewald O, Ren G, Duerr GD et al.: Of mice and dogs: species-specific differences in the inflammatory response following myocardial infarction. Am. J. Pathol. 164, 665-677 (2004).

32. Frangogiannis NG, Ren G, Dewald O et al: The critical role of endogenous thrombospondin (TSP)-1 in preventing expansion of healing myocardial infarcts. Circulation 111, 2935-2942 (2005).

33. Segers VF, Tokunou T, Higgins LJ, MacGillivray C, Gannon J, Lee RT: Local delivery of protease-resistant stromal cell derived factor-1 for stem cell recruitment after myocardial infarction. Circulation 116, 1683-1692 (2007).

34. Bujak M, Kweon HJ, Chatila K, Li N, Taffet G, Frangogiannis NG: Aging-related defects are associated with adverse cardiac remodeling in a mouse model of reperfused myocardial infarction. J. Am. Coll. Cardiol. 51, 1384-1392 (2008).

35. Fahey TJ, 3rd, Sadaty A, Jones WG 2nd, Barber A, Smoller B, Shires GT: Diabetes impairs the late inflammatory response to wound healing. J. Surg. Res. 50, 308-313 (1991).
36. Greer JJ, Ware DP, Lefer DJ: Myocardial infarction and heart failure in the db/db diabetic mouse. Am. J. Physiol. Heart Circ. Physiol. 290, H146-H153 (2006).

\section{Affiliations}

- Marcin Dobaczewski, MD Baylor College of Medicine, Section of Cardiovascular Sciences, One Baylor Plaza, BCM620, Houston, TX 77030 , USA

Tel.: +1 713798 4188;

Fax: +17137960015;

marcind@bcm.tmc.edu

- Nikolaos G Frangogiannis, MD Baylor College of Medicine, Section of Cardiovascular Sciences, One Baylor Plaza M/S F-602, Houston, TX 77030 , USA

Tel.: + 1713798 4188;

Fax: +17137960015

ngf@bcm.tmc.edu 